\title{
Constraints on scalar-tensor theory of gravity by solar system tests
}

\author{
P. A. González ${ }^{1, \mathrm{a}}$, Marco Olivares ${ }^{1, \mathrm{~b}}$, Eleftherios Papantonopoulos ${ }^{2, \mathrm{c}}$, Yerko Vásquez ${ }^{3, \mathrm{~d}}$ \\ ${ }^{1}$ Facultad de Ingeniería y Ciencias, Universidad Diego Portales, Avenida Ejército Libertador 441, Casilla 298-V, Santiago, Chile \\ 2 Department of Physics, National Technical University of Athens, Zografou Campus, 15773 Athens, Greece \\ ${ }^{3}$ Departamento de Física, Facultad de Ciencias, Universidad de La Serena, Avenida Cisternas 1200, La Serena, Chile
}

Received: 20 June 2020 / Accepted: 10 October 2020 / Published online: 22 October 2020

(C) The Author(s) 2020

\begin{abstract}
We study the motion of particles in the background of a scalar-tensor theory of gravity in which the scalar field is kinetically coupled to the Einstein tensor. We constrain the value of the derivative parameter $z$ through solar system tests. By considering the perihelion precession we obtain the constraint $\sqrt{z} / m_{\mathrm{p}}>2.6 \times 10^{12} \mathrm{~m}$, the gravitational redshift $\frac{\sqrt{z}}{m_{\mathrm{p}}}>2.7 \times 10^{10} \mathrm{~m}$, the deflection of light $\sqrt{z} / m_{\mathrm{p}}>1.6 \times 10^{11} \mathrm{~m}$, and the gravitational time delay $\sqrt{z} / m_{\mathrm{p}}>7.9 \times 10^{12} \mathrm{~m}$; thereby, our results show that it is possible to constrain the value of the $z$ parameter in agreement with the observational tests that have been considered.
\end{abstract}

\section{Contents}

1 Introduction . . . . . . . . . . . . 1

2 Four-dimensional Horndeski black hole . . . . . . . 3

3 Solar test for the Horndeski black hole . . . . . . . 4

3.1 Time-like geodesics . . . . . . . . . . . . . . . . 4

3.1 .1 Radial motion . . . . . . . . . . . . 5

3.1 .2 Angular motion . . . . . . . . . 5 5

3.1.3 Perihelion precession . . . . . . . . 6

3.2 Null geodesics . . . . . . . . . . . . . . . . . . 7

3.2.1 Radial motion . . . . . . . . . . . . . 7

3.2.2 Angular motion . . . . . . . . . . . 7

3.2.3 Gravitational redshift . . . . . . . . . 8

3.2.4 Deflection of light . . . . . . . . . 8

3.2.5 Gravitational time delay . . . . . . . . . 9 9

4 Concluding comments . . . . . . . . . . . . . . 10

References ................. . 11

a e-mail: pablo.gonzalez@udp.cl
b e-mail: marco.olivaresr@mail.udp.cl
c e-mail: lpapa@central.ntua.gr
de-mail: yvasquez@userena.cl (corresponding author)

\section{Introduction}

Modified theories of gravity were recently introduced in an attempt to cure certain inconsistencies of General Relativity (GR) and to explain certain observational results on dark matter and dark energy. These theories introduce modifications of GR on short and large distances in the attempt to provide a viable theory of gravity. The recent observational results on the Gravitational Waves (GWs) [1-5] provide a new area of testing alternative gravity theories and differentiating them from GR. Therefore, it is very important to study the compact objects predicted by different modified theories of gravity and possible GWs as regards the observational signatures they might give. It would also be very interesting to study if classical solar system tests on these objects such as light deflection, the perihelion shift of planets, and gravitational time delay can give any discrepancy between the GR and the observations.

Some of the simplest and extensively studied modifications of GR are the scalar-tensor theories [6]. The presence of a scalar field coupled to gravity results in black holes and compact objects dressed with a hairy matter distribution. The Horndeski Lagrangian [7] provides one of the best-studied scalar-tensor theories. This is because the Horndeski theories lead to second-order field equations and they result in consistent theories without ghost instabilities [8] and they preserve a classical Galilean symmetry $[9,10]$. The Horndeski theory has been studied on short and large distances. Local black hole solutions were found in a subclass of Horndeski theories which are characterized by the presence of a scalar field which is kinetically coupled to the Einstein tensor [11-15]. On large distances the presence of the derivative coupling acts as a friction term in the inflationary period of the cosmological evolution [16-22]. This derivative coupling introduces a mass scale in the theory which on large distances can be constrained by the recent results on GWs. 
If we assume that the dark energy drives the late cosmological expansion and is parameterized by a scalar field coupled to the Einstein tensor it is found [23,24] that the propagation speed of the tensor perturbations around the cosmological Friedmann-Robertson-Walker (FRW) background is different from the speed of light $c$. Therefore the measurement of the speed of GWs can be used to constrain the value of the derivative coupling and test in general the applicability of Horndeski theories at large distances [25-31].

The measurement of the speed of GWs by GW170817 and GRB170817A gave an upper bound on the speed of GWs $c_{\mathrm{gw}} / c-1 \leq 7 \times 10^{-16}$ [32]. If we assume that the peak of the GW signal and gamma ray burst GRB170817A were emitted simultaneously then we get a lower bound $c_{\mathrm{gw}} / c-1>-3 \times 10^{-15}$ [32], so we can safely conclude that $c_{\mathrm{gw}}=c$. The precise measurement of the propagation speed of GWs is a very powerful tool to constrain the applicability of the Horndeski theory. Notably, in the Horndeski theory [7] and its generalizations [33], the functions of the scalar field $\phi$ and its kinetic energy $X=-\partial_{\mu} \phi \partial^{\mu} \phi / 2, G_{4}(\phi, X)$ and $G_{5}(\phi, X)$ should be constrained in order to be consistent with the above observations. The reason is that these terms provide the kinetic energy of the scalar field coupled to gravity and they influence the speed of GWs. The term $G_{5}(\phi, X)$ is the general coupling of the scalar field to the Einstein tensor and in [34], assuming that the scalar field plays the role of dark energy, a lower bound on the mass scale present in this term was found and combining the constraints from inflation the energy scale of the derivative coupling is bounded to be $10^{15} \mathrm{GeV} \gg M \gtrsim 2 \times 10^{-35} \mathrm{GeV}$.

Modified gravity theories can also be compared to GR predictions at relative small scales. Solar system observations, such as light deflection, the perihelion shift of planets, and gravitational time delay are described within GR. To study such effects you have to calculate the geodesics for the motion of particles around a black hole background. In [35] the perihelion precession of planetary orbits and the bending angle of null geodesics are estimated for different gravity theories in string-inspired models. The solar system effects have been studied in black hole AdS geometries by calculating the motion of particles on AdS spacetime [36-43]. The motion of massless and massive particles in the background of four-dimensional asymptotically AdS black holes with scalar hair [44] were studied in [45]. The geodesics were studied numerically and the differences in the motion of particles between the four-dimensional asymptotically AdS black holes with scalar hair and their no-hair limit were discussed. In the context of solar system and astrophysical scenarios spherically symmetric solutions resulting from the coupling of the Gauss-Bonnet with a scalar field were discussed in [46].

Care should be taken when one studies specific scalartensor theories and compare their predictions with GR. In general scalar fields, depending on their coupling to gravity, mediate fifth forces. Therefore in these theories scalar fields should accommodate a mechanism to suppress the scalar interaction on small scales and make sure that precision tests of gravity at solar system scales are applicable. There are various screening mechanisms to suppress scalar interactions on small scales. One of the basic screening mechanisms is the Vainshtein mechanism [47], which was developed for the massive gravity (for an extensive review of the Vainshtein mechanism in massive gravity see [48]). The Vainshtein screening mechanism applies also to Galileon-like models [9] and to nonlinear massive gravity [49-51] in which the presence of nonlinear derivative scalar fields $\phi$ with self-interactions can suppress the propagation of fifth forces through the Vainshtein mechanism. In [52,53] the consequences of the Vainshtein mechanism were studied in scalar-tensor theories taking into account the nonlinear effect. Therefore, for models of modified gravity we need to clarify the behavior of gravity around and below the scale at which the relevant nonlinearities appear in order to test them against experiments and cosmological observations.

An extensive study of the Vainshtein mechanism was carried out in [54] in the most general scalar-tensor theories with second-order equations of motion resulting from a spherically symmetric spacetime with a matter source. One applied the general results to a number of concrete models such as the covariant/extended Galileons and the Dirac-Born-Infeld Galileons with Gauss-Bonnet and other terms. One found that in these theories the fifth force can be suppressed and be consistent with solar system constraints, provided that nonlinear field kinetic terms coupled to the Einstein tensor do not dominate over other nonlinear field self-interactions.

The aim of this work is to constrain the parameters of the subclass of the Horndeski theory with a scalar field coupled kinetically to the Einstein tensor using the solar system observations. As we have already referred to, there are various black hole solutions in this subclass of Horndeski theory. In [11] numerical black hole solutions were discussed with a scalar field coupled to the Einstein tensor. Also in [14] static and spherically symmetric black hole solutions were found if the scalar field is time dependent. For our study we have to work with an exact black hole solution with a static matter distribution outside the horizon of the black hole. Another important requirement is that the derivative coupling of the scalar field to Einstein tensor should appear in the metric function of the black hole in order to constrain this coupling from the solar system tests. A black hole solution that satisfies these constraints is the well-studied black hole solution of Horndeski theory discussed in [12].

As we already discussed, to apply the solar system test to our model it should be consistent with GR. For this to happen nonlinear field kinetic terms should not dominate the dynamics. In [54] there is an extensive study of the Horndeski 
theories in which $G_{5}(\phi, X) \neq 0$ as in the model we study. In the solution [12] the coupling function of the kinetic scalar term to Einstein tensor is constant, independent of the scalar field itself. Then it was shown in [54] that in this case we do not expect any nonlinearities to appear in our model and then GR can be recovered at small distances. In this model because of the shift symmetry the scalar field appears only through its derivative and then $\psi=\phi^{\prime}$ appears as an extra degree of freedom, expressed by the real quantity $\psi^{2}$. Following mainly the work in $[55,56]$ we will study the effects of the solar system tests by considering the perihelion precession, the gravitational redshift, the deflection of light and the gravitational time delay.

This paper is organized as follows. In Sect. 2 we give a brief review of the four-dimensional Horndeski black hole of [12], which we will consider as background. In Sect. 3 we study the motion of massless and massive particles, and we perform some classical tests such as the perihelion precession, the deflection of light and the gravitational time delay. Finally, in Sect. 4 we conclude.

\section{Four-dimensional Horndeski black hole}

In this section after reviewing Horndeski theory we will discuss a particular hairy black hole solution [12] of the Horndeski theory generated by a scalar field non-minimally coupled to Einstein tensor.

The action of the Horndeski theory is given by [7],

$S=\int \mathrm{d}^{4} x \sqrt{-g}\left(L_{2}+L_{3}+L_{4}+L_{5}\right)$,

where

$$
\begin{aligned}
L_{2}= & K(\phi, X), \quad L_{3}=-G_{3}(\phi, X) \square \phi, \\
L_{4}= & G_{4}(\phi, X) R+G_{4, X}\left[(\square \phi)^{2}-\left(\nabla_{\mu} \nabla_{\nu} \phi\right)\left(\nabla^{\mu} \nabla^{v} \phi\right)\right], \\
L_{5}= & G_{5}(\phi, X) G_{\mu \nu} \nabla^{\mu} \nabla^{v} \phi-\frac{1}{6} G_{5, X}\left[(\square \phi)^{3}\right. \\
& -3(\square \phi)\left(\nabla_{\mu} \nabla_{\nu} \phi\right)\left(\nabla^{\mu} \nabla^{v} \phi\right) \\
& \left.+2\left(\nabla^{\mu} \nabla_{\alpha} \phi\right)\left(\nabla^{\alpha} \nabla_{\beta} \phi\right)\left(\nabla^{\beta} \nabla_{\mu} \phi\right)\right],
\end{aligned}
$$

with $X=-\nabla_{\mu} \phi \nabla^{\mu} \phi / 2, \square \phi=\nabla_{\mu} \nabla^{\mu} \phi$, and where the functions $K, G_{3}, G_{4}$ and $G_{5}$ are arbitrary functions of $\phi$ and $X$, and $G_{j, X}(\phi, X)=\partial G_{j}(\phi, X) / \partial X$ with $j=4,5$.

This action is the most general one for scalar-tensor theory with at most second-order field equations. If we take $K=$ $G_{3}=G_{5}=0$ and $G_{4}=M_{\mathrm{Pl}} / 2$, then we obtain Einstein's general relativity. If we take $G_{3}=G_{5}=0, K=X-V(\phi)$, and $G_{4}=f(\phi)$, then we get scalar-tensor $f(\phi) R$ theories. If we take $G_{4}=M_{\mathrm{Pl}}^{2} / 2+X /\left(2 M^{2}\right)$ or $G_{4}=M_{\mathrm{Pl}}^{2} / 2$ and
$G_{5}=-\phi /\left(2 M^{2}\right)$, then we get the non-minimally derivative coupling $G_{\mu \nu} \nabla^{\mu} \phi \nabla^{\nu} \phi /\left(2 M^{2}\right)$ with the mass scale $M$.

As can be seen in the action (1) of Horndeski theory, except the minimal coupling of the scalar field to gravity, there are other higher order couplings of the scalar field and also a term contained in the Lagrangian $L_{5}$ of the scalar field directly coupled to the Einstein tensor. This term is interesting because it gives information of how strongly matter is coupled to curvature. Therefore, following the discussion of the introduction, it would be interesting to see how this coupling, except for the constraints it has from the GWs, is further constrained by the solar system tests.

We will only consider the non-minimal derivative coupling of the scalar field to the Einstein tensor of the Horndeski theory given by the Lagrangian

$\mathfrak{L}=\frac{m_{\mathrm{p}}^{2}}{2} R-\frac{1}{2}\left(g^{\mu \nu}-\frac{z}{m_{\mathrm{p}}^{2}} G^{\mu \nu}\right) \partial_{\mu} \phi \partial_{\nu} \phi$,

where $m_{\mathrm{p}}$ is the Planck mass, $z$ is the derivative coupling of the scalar field to Einstein tensor, $G_{\mu \nu}$ the Einstein tensor, $\varphi$ a scalar field, and $g_{\mu \nu}$ is the metric. The absence of scalar potential allows for the shift symmetry $\varphi \rightarrow \varphi+$ const, which is the relevant Galileon symmetry that survives in curved space.

Consider the metric ansatz

$$
\mathrm{d} s^{2}=-F(r) \mathrm{d} t^{2}+G(r) \mathrm{d} r^{2}+\rho^{2}(r)\left(\mathrm{d} \theta^{2}+\sin ^{2}(\theta) \mathrm{d} \phi^{2}\right) .
$$

Setting $\rho=r$ the equations of motion are [12]

$r \frac{F^{\prime}}{F}=G-1+\frac{m_{\mathrm{p}}^{2} r^{2} G}{z}+\frac{K m_{\mathrm{p}}^{2} G^{2}}{z \psi \sqrt{F G}}$,

$r \frac{F^{\prime}}{F}=\frac{2 m_{\mathrm{p}}^{4} G(G-1)+z \psi^{2}(G-3)+m_{\mathrm{p}}^{2} r^{2} G \psi^{2}}{\left(3 z \psi^{2}+2 m_{\mathrm{p}}^{4} G\right)}$,

$\frac{r}{2}\left(\frac{F^{\prime}}{F}-\frac{G^{\prime}}{G}\right)=\frac{2 m_{\mathrm{p}} G(G-1)-2 z \psi^{2}-z r\left(\psi^{2}\right)^{\prime}}{\left(3 z \psi^{2}+2 m_{\mathrm{p}}^{4} G\right)}$,

where $K$ is an integration constant and $\psi \equiv \varphi^{\prime}$. We can see that $\psi=0$ implies $K=0$ and the resulting metric turns out to be the Schwarzschild one. When $K=0$ and $z \neq 0$, analytical exact solutions of the system were found which depend on the sign of $z$ and to avoid nonphysical modes for the scalar field $z>0$ was considered,

$$
\begin{aligned}
F(r) & =\frac{3}{4}+\frac{r^{2} m_{\mathrm{p}}^{2}}{12 z}-\frac{2 M}{m_{\mathrm{p}}^{2} r}+\frac{\sqrt{z}}{4 m_{\mathrm{p}} r} \arctan \left(\frac{m_{\mathrm{p}} r}{\sqrt{z}}\right), \\
G(r) & =\frac{\left(m_{\mathrm{p}}^{2} r^{2}+2 z\right)^{2}}{4\left(m_{\mathrm{p}}^{2} r^{2}+z\right)^{2} F(r)}, \\
\psi^{2}(r) & =-\frac{m_{\mathrm{p}}^{6} r^{2}\left(m_{\mathrm{p}}^{2} r^{2}+2 z\right)^{2}}{4 z\left(m_{\mathrm{p}}^{2} r^{2}+z\right)^{3} F(r)},
\end{aligned}
$$


where $l^{2}=12 z / m_{\mathrm{p}}^{2}$ and $M$ is a constant of integration that will play the role of a mass. As discussed in [12] $z$ is a nonperturbative parameter when we regard the Lagrangian (2) as a theory of modified gravity. Indeed, the deviation from GR vanishes when $z$ diverges and the scalar field is strongly coupled. Also, the parameter $z$ clearly interpolates between the flat black hole solution and the Schwarzschild AdS one as $1 / z$ essentially plays the role of an effective negative cosmological constant.

\section{Solar test for the Horndeski black hole}

In order to find the effects of the solar system to the Horndeski black hole we have to study the geodesics of the spacetime described by (3). For this purpose, we will solve the EulerLagrange equations for the variational problem associated with this metric. The Lagrangian associated with the metric (3) is given by

$2 \mathcal{L}=-F(r) \dot{t}^{2}+G(r) \dot{r}^{2}+r^{2}\left(\dot{\theta}^{2}+\sin ^{2} \theta \dot{\phi}^{2}\right)=-m$,

where $\dot{q}=\mathrm{d} q / \mathrm{d} \tau$, and $\tau$ is an affine parameter along the geodesic. Since the Lagrangian (10) is independent of the cyclic coordinates $(t, \phi)$, their conjugate momenta $\left(\Pi_{t}, \Pi_{\phi}\right)$ are conserved and the equations of motion read

$\dot{\Pi}_{q}-\frac{\partial \mathcal{L}}{\partial q}=0$,

where $\Pi_{q}=\partial \mathcal{L} / \partial \dot{q}$ is the conjugate momentum of the coordinate $q$. Using (10), the above equation yields

$$
\begin{gathered}
\dot{\Pi}_{t}=0, \quad \dot{\Pi}_{r}=-\frac{\dot{t}^{2}}{2} \frac{\mathrm{d} F(r)}{\mathrm{d} r}+\frac{\dot{r}^{2}}{2} \frac{\mathrm{d} G(r)}{\mathrm{d} r} \\
+r\left(\dot{\theta}^{2}+\sin ^{2} \theta \dot{\phi}^{2}\right), \\
\dot{\Pi}_{\theta}=r^{2} \sin \theta \cos \theta \dot{\phi}^{2}, \quad \text { and } \quad \dot{\Pi}_{\phi}=0,
\end{gathered}
$$

and the conjugate momenta are given by

$$
\begin{aligned}
\Pi_{t} & =-F(r) \dot{t}, \quad \Pi_{r}=G(r) \dot{r}, \\
\Pi_{\theta} & =r^{2} \dot{\theta}, \quad \text { and } \quad \Pi_{\phi}=r^{2} \sin ^{2} \theta \dot{\phi} .
\end{aligned}
$$

Now, without loss of generality, we consider the motion to develop in the invariant plane $\theta=\pi / 2$ and $\dot{\theta}=0$, which is characteristic of central fields. With this choice, Eqs. (13) and (14) become

$$
\Pi_{t}=-F(r) \dot{t} \equiv-\sqrt{E}, \quad \Pi_{\phi}=r^{2} \dot{\phi} \equiv L,
$$

where $E$ and $L$ are integration constants associated with each of the expressions. So, inserting Eq. (15) into Eq. (10) we obtain

$\left(\frac{\mathrm{d} r}{\mathrm{~d} \tau}\right)^{2}=\frac{E-V(r)}{F(r) G(r)}$,

where $V(r)$ is the effective potential given by

$V(r)=F(r)\left[m+\frac{L^{2}}{r^{2}}\right]$,

where $m$ is the test mass, and by normalization we shall take $m=1$ for massive particles and $m=0$ for photons. Finally, using (15) and (16) we obtain the following equations:

$$
\begin{aligned}
& \left(\frac{\mathrm{d} r}{\mathrm{~d} t}\right)^{2}=\frac{F(r)}{E}\left(\frac{E-V(r)}{G(r)}\right), \\
& \left(\frac{\mathrm{d} r}{\mathrm{~d} \phi}\right)^{2}=\frac{r^{4}}{L^{2}}\left(\frac{E-V(r)}{F(r) G(r)}\right) .
\end{aligned}
$$

In the following we will consider the regime $r<$ $\sqrt{z} / m_{\mathrm{p}}$. Thus, for $0<m_{p} r / \sqrt{z}<1 \arctan m_{p} r / \sqrt{z} \approx$ $\sum_{n=0}^{\infty} \frac{(-1)^{n}}{2 n+1}\left(m_{p} r / \sqrt{z}\right)^{2 n+1}$. Therefore, the lapse function Eq. (7) can be written as

$F(r) \approx \frac{3}{4}+\frac{r^{2} m_{\mathrm{p}}^{2}}{12 z}-\frac{2 M}{m_{\mathrm{p}}^{2} r}+\frac{\sqrt{z}}{4 m_{\mathrm{p}} r} \sum_{j=0}^{\infty} \frac{(-1)^{j}}{2 j+1}\left(\frac{m_{\mathrm{p}} r}{\sqrt{z}}\right)^{2 j+1}$.

Now, by considering the first three terms of the summation, we obtain

$F(r) \approx 1-\frac{2 M}{m_{\mathrm{p}}^{2} r}+\frac{r^{4} m_{\mathrm{p}}^{4}}{20 z^{2}}, \quad G(r) \approx \frac{1}{F(r)}$.

Note that the lapse function approximates the Schwarzschild case when $z \rightarrow \infty$, and $m_{\mathrm{p}}=1$. With this approximation, the event horizon corresponds to the real solution of $F(r)=0$, given by

$$
\begin{aligned}
r_{+} & =\frac{2 M}{m_{\mathrm{p}}^{2}} 4 F_{3} \\
\times & \times\left[\left\{\frac{1}{5}, \frac{2}{5}, \frac{3}{5}, \frac{4}{5}\right\} ;\left\{\frac{1}{2}, \frac{3}{4}, \frac{5}{4}\right\} ; \frac{3125}{256}\left(-\frac{40 M z^{2}}{m_{\mathrm{p}}\left(-20 z^{2}\right)^{5 / 4}}\right)^{4}\right],
\end{aligned}
$$

where ${ }_{4} F_{3}\left[\left\{a_{1}, a_{2}, a_{3}, a_{4}\right\},\left\{b_{1}, b_{2}, b_{3}\right\}, x\right]$ is the generalized hypergeometric function.

\subsection{Time-like geodesics}

In order to observe the possible orbits, we plot the effective potential for massive particles (17) which is shown in Fig. 1. In the following, we describe the radial motion and the angular motion. 

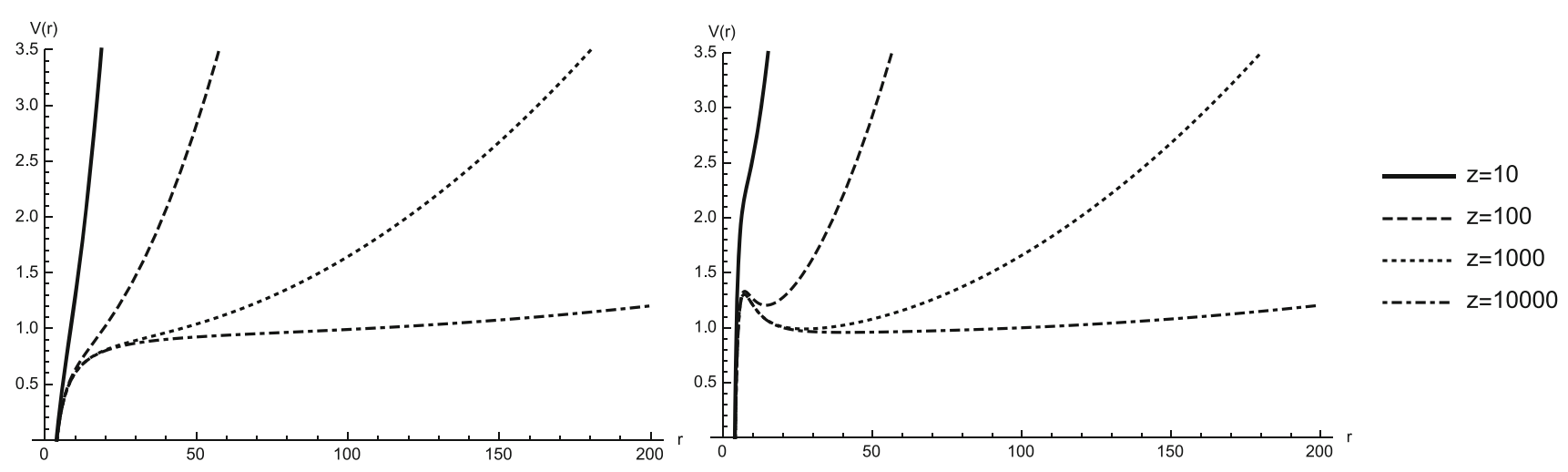

Fig. 1 The behavior of $V(r)$ for radial particles $(L=0)$ left panel, and non-radial particles $(L=10)$ right panel, as a function of $r$, with $M=2$, and $m_{\mathrm{p}}=1$

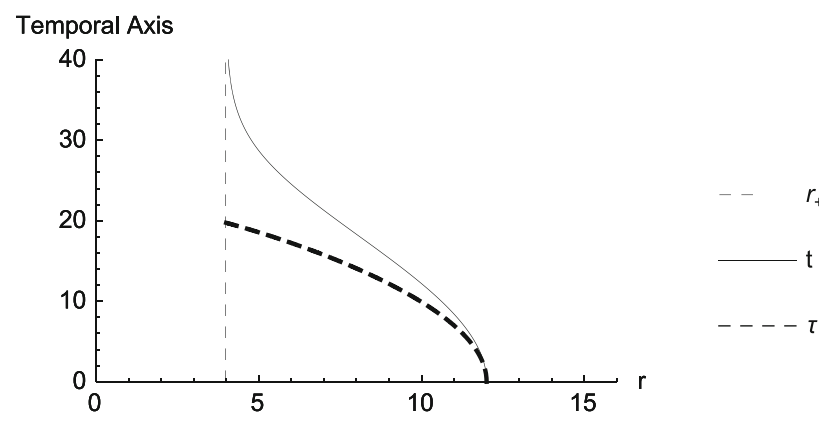

Fig. 2 The behavior of the proper $(\tau)$ and the coordinate $(t)$ time as a function of $r$, with $z=100, R_{0}=12$, and $V\left(R_{0}\right)=0.719$

\subsubsection{Radial motion}

In this case $L=0$. The particles always fall into the horizon from an upper distance $R_{0}$. Note that the proper time $(\tau)$ depends on the energy of the test particle, while the coordinate time $(t)$ does not depend on the energy of the test particle. In Fig. 2 we plot the proper time $(\tau)$ and the coordinate time $(t)$ as a function of $r$ for a particle falling from a finite distance with zero initial velocity; we see that the particle falls towards the horizon in a finite proper time. The situation is very different if we consider the trajectory in the coordinate time, where $t$ goes to infinity. This physical result is consistent with the Schwarzschild black hole.

\subsubsection{Angular motion}

For the angular motion we consider $L>0$. The allowed orbits depend on the value of the constant $E$.

- If $E=E_{\mathrm{s}} \approx 1.204$ the particle can orbit in a stable circular orbit at $r_{\mathrm{s}}=14.230$; see Fig. 3 .

- If $E=E_{\mathrm{u}} \approx 1.331$ the particle can orbit in an unstable circular orbit at $r_{\mathrm{u}}=7.126$. Also, there are two critical orbits that approximate asymptotically the unstable

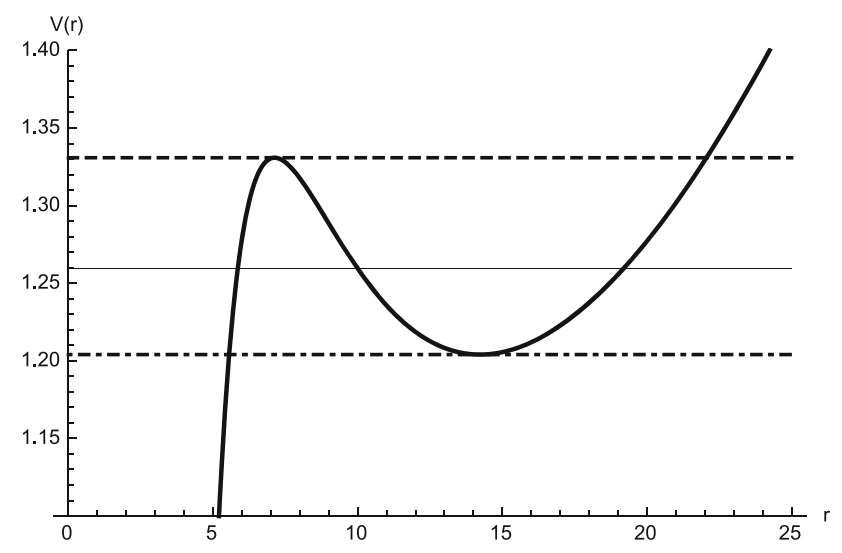

Fig. 3 The behavior of $V(r)$ for non-radial particles $(L=10)$ as a function of $r$, with $M=2, z=100$, and $m_{\mathrm{p}}=1$. The dashed line corresponds to $E=E_{\mathrm{u}} \approx 1.331$, the dot dashed line corresponds to $E=E_{\mathrm{s}} \approx 1.204$ and the thin line corresponds to $E=1.259$

circular orbit. For the first kind orbit the particle starts from rest and at a finite distance greater than the unstable radius. For the second kind orbit the particle starts from a finite distance greater than the horizon, but smaller than the unstable radius.

- The planetary orbits are constrained to oscillate between an apastron and a periastron. We plot in Fig. 4 the planetary orbit for $E=1.259$. We can observe that the particle completes an oscillation in an angle greater than $2 \pi$ which is similar to the Schwarzschild black hole [57].

It is possible to calculate the periods of the circular orbits $\left(r_{\text {c.o. }}\right)$, which can be stable $\left(r_{\mathrm{s}}\right)$ or unstable $\left(r_{\mathrm{u}}\right)$ orbits using the constants of motion $\sqrt{E}$ and $L$, given by (15), which yields

$T_{\tau}=2 \pi \sqrt{\frac{2 r_{\text {c.o. }} F\left(r_{\text {c.o. }}\right)-r_{\text {c.o. }}^{2} F^{\prime}\left(r_{\text {c.o. }}\right)}{F^{\prime}\left(r_{\text {c.o. }}\right)}}$ 


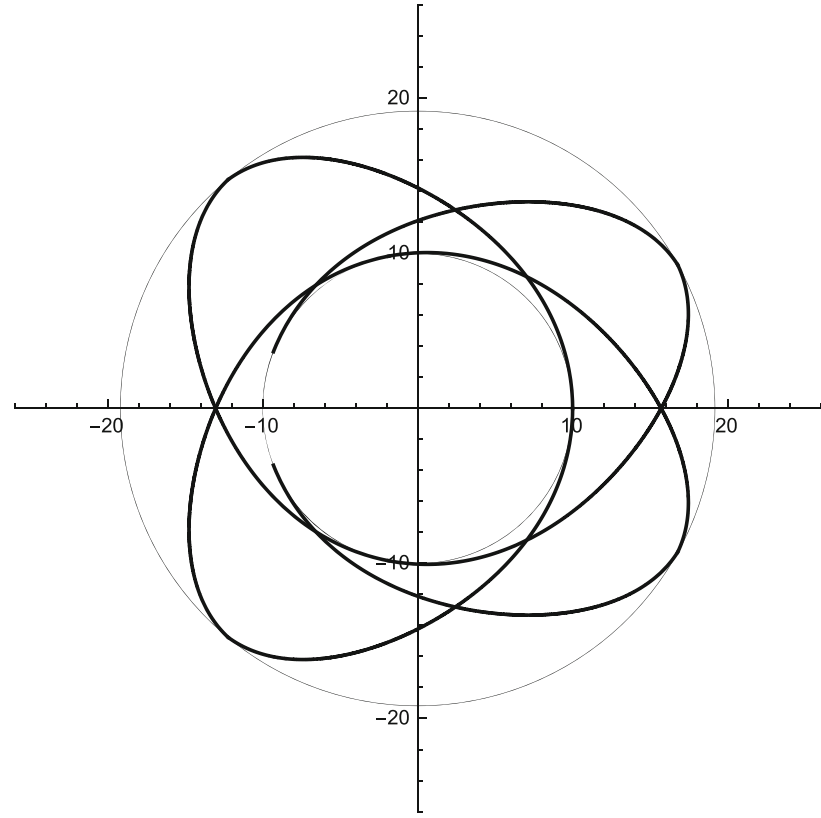

Fig. 4 The planetary orbit for $L=10, r_{\mathrm{A}}=19.185, r_{\mathrm{P}}=10$ and $E=1.259$

and

$T_{t}=2 \pi \sqrt{\frac{2 r_{\text {c.o. }}}{F^{\prime}\left(r_{\text {c.o. }}\right)}}$

where $T_{\tau}$ is the period of the orbit with respect to the proper time and $T_{t}$ is the period of the orbit with respect to the coordinate time. It is worth to mention that the periods depend on the lapse function and the derivatives, which contain the $M$ and $z$ parameter. On the other hand, for the stable circular orbits it is possible to find the epicycle frequency, given by $\kappa^{2}=V^{\prime \prime}\left(r_{\mathrm{s}}\right) / 2$, which yields

$\kappa^{2}=\frac{F^{\prime \prime}\left(r_{\mathrm{s}}\right)}{2}+\frac{F^{\prime}\left(r_{\mathrm{s}}\right)}{2}\left(\frac{r_{\mathrm{s}}^{2} F^{\prime \prime}\left(r_{\mathrm{s}}\right)-4 r_{s} F^{\prime}\left(r_{\mathrm{s}}\right)+6 F\left(r_{\mathrm{s}}\right)}{2 r_{\mathrm{s}} F\left(r_{\mathrm{s}}\right)-r_{\mathrm{s}}^{2} F^{\prime}\left(r_{\mathrm{s}}\right)}\right)$.

\subsubsection{Perihelion precession}

The following treatment, performed by Cornbleet [58], allows us to derive the formula for the advance of the perihelia of planetary orbits. The starting point is to consider the line element in unperturbed Lorentz coordinates,

$\mathrm{d} s^{2}=-\mathrm{d} t^{2}+\mathrm{d} r^{2}+r^{2}\left(\mathrm{~d} \theta^{2}+\sin ^{2} \theta \mathrm{d} \phi^{2}\right)$,

together with line element (3), where $F(r)$ and $G(r)$ are given by (21). So, considering only the radial and time coordinates in the binomial approximation, the transformation gives

$$
\begin{aligned}
& \mathrm{d} \tilde{t} \approx\left(1-\frac{M}{m_{\mathrm{p}}^{2} r}+\frac{r^{4} m_{\mathrm{p}}^{4}}{40 z^{2}}\right) \mathrm{d} t, \\
& \mathrm{~d} \tilde{r} \approx\left(1+\frac{M}{m_{\mathrm{p}}^{2} r}-\frac{r^{4} m_{\mathrm{p}}^{4}}{40 z^{2}}\right) \mathrm{d} r .
\end{aligned}
$$

We will consider two elliptical orbits: the classical Kepler orbit in $(r, t)$ space and a Horndeski orbit in $(\tilde{r}, \tilde{t})$ space. Then, in the Lorentz space $\mathrm{d} A=\int_{0}^{\mathcal{R}} r \mathrm{~d} r \mathrm{~d} \phi=\mathcal{R}^{2} \mathrm{~d} \phi / 2$, and

$\frac{\mathrm{d} A}{\mathrm{~d} t}=\frac{1}{2} \mathcal{R}^{2} \frac{\mathrm{d} \phi}{\mathrm{d} t}$

which corresponds to Kepler's second law. For the Horndeski case we have

$\mathrm{d} \tilde{A}=\int_{0}^{\mathcal{R}} r \mathrm{~d} \tilde{r} \mathrm{~d} \phi$

where $\mathrm{d} \tilde{r}$ is given by Eq. (28). So, we can write (30) as

$$
\begin{aligned}
\mathrm{d} \tilde{A} & =\int_{0}^{\mathcal{R}} r\left(1+\frac{M}{m_{\mathrm{p}}^{2} r}-\frac{r^{4} m_{\mathrm{p}}^{4}}{40 z^{2}}\right) \mathrm{d} r \mathrm{~d} \phi \\
& \approx \frac{\mathcal{R}^{2}}{2}\left(1+\frac{2 M}{m_{\mathrm{p}}^{2} \mathcal{R}}-\frac{\mathcal{R}^{4} m_{\mathrm{p}}^{4}}{120 z^{2}}\right) \mathrm{d} \phi
\end{aligned}
$$

Therefore, applying the binomial approximation wherever necessary, we obtain

$$
\begin{aligned}
\frac{\mathrm{d} \tilde{A}}{\mathrm{~d} \tilde{t}}= & \frac{\mathcal{R}^{2}}{2}\left(1+\frac{2 M}{m_{\mathrm{p}}^{2} \mathcal{R}}-\frac{\mathcal{R}^{4} m_{\mathrm{p}}^{4}}{120 z^{2}}\right) \frac{\mathrm{d} \phi}{\mathrm{d} \tilde{t}} \\
\approx & \frac{\mathcal{R}^{2}}{2}\left(1+\frac{2 M}{m_{\mathrm{p}}^{2} \mathcal{R}}-\frac{\mathcal{R}^{4} m_{\mathrm{p}}^{4}}{120 z^{2}}\right) \\
& \times\left(1+\frac{M}{m_{\mathrm{p}}^{2} \mathcal{R}}-\frac{\mathcal{R}^{4} m_{\mathrm{p}}^{4}}{40 z^{2}}\right) \frac{\mathrm{d} \phi}{\mathrm{d} t} .
\end{aligned}
$$

So, using this increase to improve the elemental angle from $d \phi$ to $d \tilde{\phi}$, then for a single orbit

$\int_{0}^{\Delta \tilde{\phi}} \mathrm{d} \tilde{\phi}=\int_{0}^{\Delta \phi=2 \pi}\left(1+\frac{3 M}{m_{\mathrm{p}}^{2} \mathcal{R}}-\frac{\mathcal{R}^{4} m_{\mathrm{p}}^{4}}{30 z^{2}}\right) \mathrm{d} \phi$

where we have neglected products of $M$ and $z$. The polar form of an ellipse is given by

$\mathcal{R}=\frac{l}{1+\epsilon \cos \phi}$ 
where $\epsilon$ is the eccentricity and $l$ is the semi-latus rectum. In this way, plugging Eq. (34) into Eq. (33), we obtain

$$
\begin{aligned}
\Delta \tilde{\phi}= & 2 \pi+\frac{3 M}{m_{\mathrm{p}}^{2}} \int_{0}^{2 \pi} \frac{1+\epsilon \cos \phi}{l} \mathrm{~d} \phi-\frac{m_{\mathrm{p}}^{4}}{30 z^{2}} \int_{0}^{2 \pi} \\
& \times\left(\frac{l}{1+\epsilon \cos \phi}\right)^{4} \mathrm{~d} \phi,
\end{aligned}
$$

which at first order yields

$\Delta \tilde{\phi} \approx 2 \pi+\frac{6 \pi M}{m_{\mathrm{p}}^{2} l}-\frac{\pi m_{\mathrm{p}}^{4} l^{4}}{15 z^{2}}$.

Note that, if we consider the limit $\frac{M}{m_{\mathrm{p}}^{2}} \rightarrow M_{\odot}$ and $z \rightarrow \infty$, we recover the classical result for the Schwarzschild spacetime. Therefore, the perihelion advance has the standard value of GR plus the correction term coming from Horndeski's theory. It is worth to mention that the observational value of the precession of perihelion for Mercury is $\Delta \tilde{\phi}_{\text {Obs. }}=5599.74,(\operatorname{arcsec} / J u l i a n-c e n t u r y) ~[59]$, and the total is $\Delta \tilde{\phi}_{\text {Total }}=5603.24$, (arcsec/Julian-century) $[60$ 64], with a difference between them of $\Delta \tilde{\phi}=-3.50$, (arcsec/Julian-century), which it is possible to attribute to a correction coming from a scalar-tensor theory, in particular coming from the parameter $z$ of Horndeski's theory $(\Delta \tilde{\phi}=$ $\left.-\frac{\pi m_{\mathrm{p}}^{4} l^{4}}{15 z^{2}}\right)$, giving the constraint $\sqrt{z} / m_{\mathrm{p}} \geq 2.6 \times 10^{12}(\mathrm{~m})$, which allows for a better accuracy between the observational value and the theoretical value of the precession of perihelion for Mercury.

\subsection{Null geodesics}

In the next analysis, we consider two kinds of motion: radial motion $(L=0)$ and angular motion $(L>0)$ of the photons $(m=0)$.

\subsubsection{Radial motion}

In this case, the master equation (16) can be written as

$\frac{\mathrm{d} r}{\mathrm{~d} \tau}= \pm \sqrt{E}$,

where $(+)$ stands for outgoing photons and $(-)$ stands for ingoing photons. The solution of the above equation yields

$r= \pm \sqrt{E} \tau+r_{0}$

where $r_{0}$ is an integration constant that corresponds to the initial position of the photon, as in the Schwarzschild case. The photons always fall into the horizon from an upper distance. In Fig. 5 we plot the affine $(\tau)$ and coordinate $(t)$ time as a function of $r$ for a photon falling from a finite distance $\left(r_{0}=12\right)$; we see that photons fall towards the horizon in a

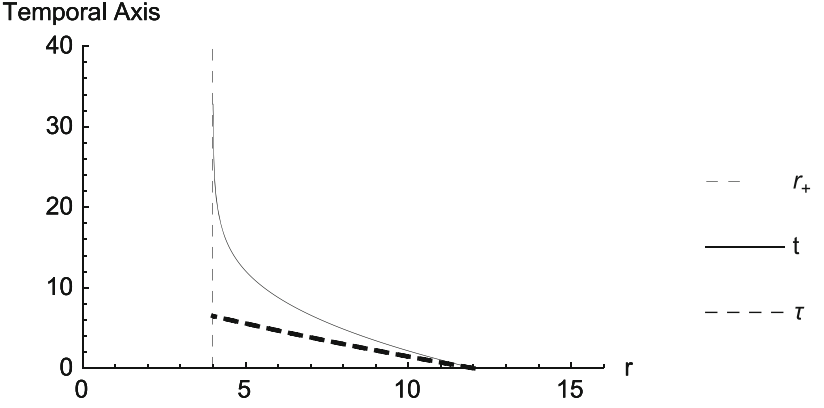

Fig. 5 The behavior of the affine $(\tau)$ and the coordinate $(t)$ time as a function of $r$, with $z=100$ and $E=1$

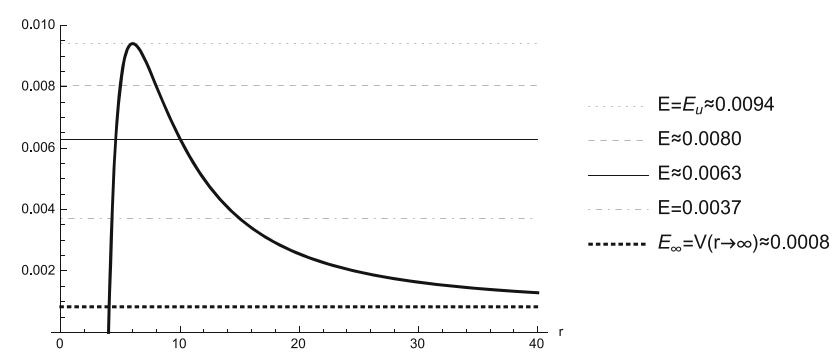

Fig. 6 The behavior of the effective potential for photons as a function of $r$, for $L=1, M=2, m_{\mathrm{p}}=1$, and $z=100$

finite affine time. The situation is very different if we consider the trajectory in the coordinate time, where $t$ goes to infinity.

\subsubsection{Angular motion}

In this case, the allowed orbits for photons depend on the value of the impact parameter $b \equiv L / \sqrt{E}$. Next, based on the impact parameter values shown in Fig. 6 , where $E_{\mathrm{u}}$ is the energy of the unstable circular orbit and $E_{\infty}=V(r \rightarrow$ $\infty)=\frac{L^{2} m_{\mathrm{p}}^{2}}{12 z}$, we give a brief qualitative description of the allowed angular motions for photons, described in the following.

- Capture zone: If $0<b<b_{\mathrm{u}}$, photons fall inexorably to the horizon, and their cross section, $\sigma$, in this geometry is [65]

$\sigma=\pi b_{\mathrm{u}}^{2}$

- Critical trajectories: If $b=b_{\mathrm{u}}\left(E_{\mathrm{u}} \approx 0.009\right)$, photons can stay in one of the unstable inner circular orbit of radius $r_{\mathrm{u}}\left(r_{\mathrm{u}} \approx 6.03\right)$. Therefore, photons that arrive from the initial distance $r_{i}\left(r_{+}<r_{i}<r_{\mathrm{u}}\right.$, or $\left.r_{\mathrm{u}}<r_{i}<\infty\right)$ can fall asymptotically into a circle of radius $r_{\mathrm{u}}$. The period with respect to the affine parameter $(\tau)$ for the unstable 


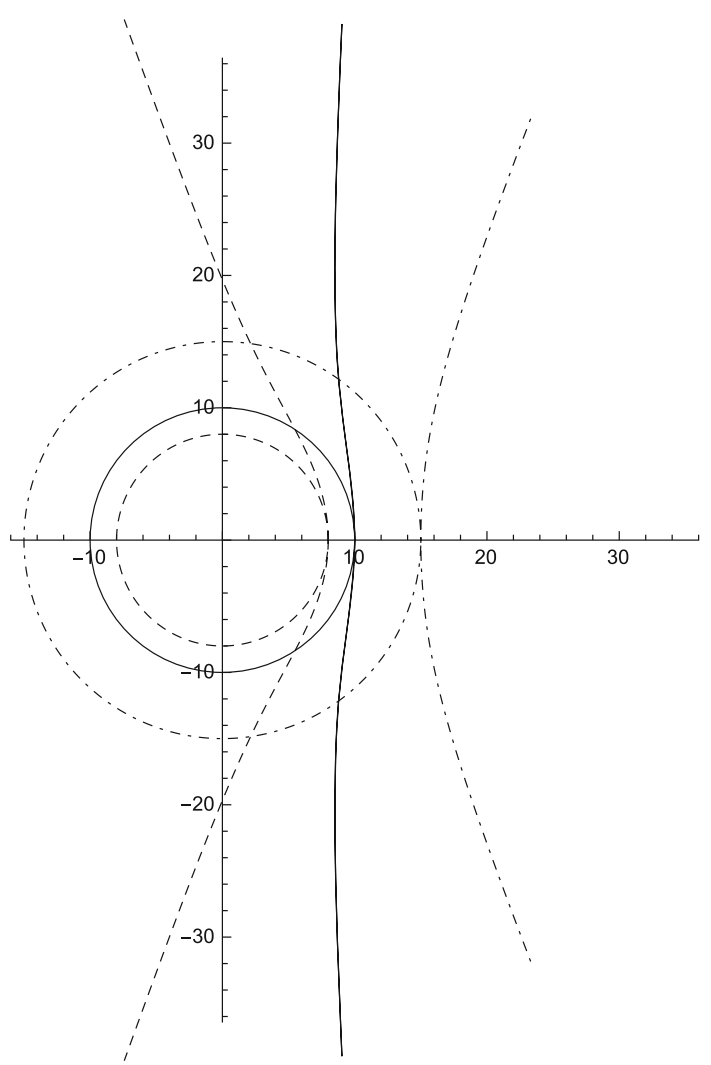

Fig. 7 The deflection of light for $L=1$ and $z=100$. The dashed line corresponds to $E=0.0080$ with $r_{\mathrm{D}}=8$, the continuous line corresponds to $E=0.0063$ with $r_{\mathrm{D}}=10$ and the dot-dashed line corresponds to $E=0.0037$ with $r_{\mathrm{D}}=15$

circular orbit is

$T_{\tau}=\frac{2 \pi r_{\mathrm{u}}^{2}}{L}$.

Also, the coordinate period is given by

$$
T_{t}=2 \pi b_{\mathrm{u}} .
$$

- Deflection zone: If $b_{\mathrm{u}}<b<b_{0} \equiv L / \sqrt{E_{\infty}}$, photons can fall from infinity to a minimum distance $r_{\mathrm{D}}$ and return to infinity. These photons are deflected; see Fig. 7. Also, we can observe a zone where the deflection is attractive and another one being repulsive. The other allowed orbits correspond to photons moving into the other side of the potential barrier, which plunge into the singularity.

\subsubsection{Gravitational redshift}

Since the Horndeski black hole is a stationary spacetime there is a time-like Killing vector so that in coordinates adapted to the symmetry the ratio of the measured frequency of a light ray crossing different positions is given by $\frac{v}{\nu_{0}}=\sqrt{\frac{g_{00}(r)}{g_{00}\left(r_{0}\right)}}$

for $M /\left(m_{\mathrm{p}}^{2} r\right) \ll 1$ and $r m_{\mathrm{p}} / \sqrt{z} \ll 1$, we obtain

$\frac{v}{\nu_{0}} \approx 1+\frac{M}{m_{\mathrm{p}}^{2} r_{0}}-\frac{M}{m_{\mathrm{p}}^{2} r}+\frac{r^{4} m_{\mathrm{p}}^{4}}{40 z^{2}}-\frac{r_{0}^{4} m_{\mathrm{p}}^{4}}{40 z^{2}}$,

where we neglected products of $z$ and $M$. Obviously, if we consider the limit $\frac{M}{m_{\mathrm{D}}^{2}} \rightarrow M_{\oplus}$ and $z \rightarrow \infty$, we recover the classical result for the Schwarzschild spacetime. The clock can be compared with an accuracy of $10^{-15}$, the H-maser in the GP-A redshift experiment [66] reaching an accuracy of $10^{-14}$. Therefore, by considering that all observations are well described within Einstein's theory, we conclude that the extra Horndeski terms must be $<10^{-14}$. Thus,

$\frac{\sqrt{z}}{m_{\mathrm{p}}}>2.7 \times 10^{10} \mathrm{~m}$

where we assume a clock comparison between Earth and a satellite at 15,000 km height, as in Ref. [67].

\subsubsection{Deflection of light}

Currently, the light-deflection phenomenon is important in modern astronomy because of its application to gravitational lenses. In order to study the bending of light, we consider Eq. (19) for photons, which can be written as

$\left(\frac{\mathrm{d} r}{\mathrm{~d} \phi}\right)^{2}=\frac{r^{4}}{b^{2}}-r^{2}+\frac{2 M}{m_{\mathrm{p}}^{2}} r-\frac{m_{\mathrm{p}}^{4}}{20 z^{2}} r^{6}$,

where $b$ is the impact parameter, and we have used Eq. (21). Now, by performing the change of variables $r=1 / u$, the above equation yields

$\left(\frac{\mathrm{d} u}{\mathrm{~d} \phi}\right)^{2}=\frac{1}{b^{2}}-u^{2}+\frac{2 M}{m_{\mathrm{p}}^{2}} u^{3}-\frac{m_{\mathrm{p}}^{4}}{20 z^{2}} u^{-2}$.

Notice that, for $z \rightarrow \infty$ and $m_{\mathrm{p}}=1$, the above equation is reduced to the classical equation of Schwarzschild for the motion of photons given by

$\left(\frac{\mathrm{d} u}{\mathrm{~d} \phi}\right)^{2}=\frac{1}{b^{2}}-u^{2}+2 M u^{3}$.

So, the derivative of Eq. (46) with respect to $\phi$ yields

$u^{\prime \prime}+u=\frac{3 M}{m_{\mathrm{p}}^{2}} u^{2}+\frac{m_{\mathrm{p}}^{4}}{20 z^{2}} u^{-3}$, 
where' denotes the derivative with respect to $\phi$. So, following the procedure established in Ref. [68], we obtain

$$
\begin{aligned}
u= & \frac{1}{b} \sin (\phi)+\frac{3 M}{2 m_{\mathrm{p}}^{2} b^{2}}+\frac{m_{\mathrm{p}}^{4} b^{3} \sqrt{2}}{10 z^{2}} \\
& +\left(\frac{M}{2 m_{\mathrm{p}}^{2} b^{2}}-\frac{m_{\mathrm{p}}^{4} b^{3} \sqrt{2}}{20 z^{2}}\right) \cos (2 \phi) .
\end{aligned}
$$

In the limit $u \rightarrow 0, \phi$ approaches $\phi_{\infty}$, with

$$
-\phi_{\infty}=\frac{2 M}{m_{\mathrm{p}}^{2} b}+\frac{m_{\mathrm{p}}^{4} b^{4} \sqrt{2}}{20 z^{2}}
$$

Therefore, for Horndeski black holes the deflection of light $\hat{\alpha}$ is equal to $2\left|-\phi_{\infty}\right|$ and yields

$\hat{\alpha}=\frac{4 M}{m_{\mathrm{p}}^{2} b}+\frac{m_{\mathrm{p}}^{4} b^{4} \sqrt{2}}{10 z^{2}}$.

Note that, if we consider the limit $\frac{M}{m_{\mathrm{p}}^{2}} \rightarrow M_{\odot}$ and $z \rightarrow \infty$, we recover the classical result of GR; that is, $\hat{\alpha}_{\mathrm{GR}}=4 M_{\odot} / b$. If the impact parameter is equal to the radius of the sun, the value obtained is $\hat{\alpha}_{\mathrm{GR}}=4 M_{\odot} / R_{\odot}=1.75092^{\prime \prime}$. The parameterized post-Newtonian (PPN) formalism introduces the phenomenological parameter $\gamma$, which characterizes the contribution of space curvature to gravitational deflection. In this formalism the deflection angle is $\hat{\alpha}=0.5(1+\gamma) 1.7426$, and currently $\gamma=0.9998 \pm 0.0004$ [69]. So, $\hat{\alpha}=1.74277^{\prime \prime}$ for $\gamma=0.9998+0.0004$ and $\hat{\alpha}=1.74208^{\prime \prime}$ for $\gamma=$ $0.9998-0.0004$. The observational values, compared to the classic result, are smaller, and the contribution of the Horndeski term to the deflection angle is positive, therefore, there is no observable effect. Thus, if the Horndeski term contributes it does so in such a way that $\hat{\alpha}_{\text {Horndeski }}<0.00001^{\prime \prime}$, or $\sqrt{z} / m_{\mathrm{p}}>1.6 \times 10^{11}(\mathrm{~m})$.

\subsubsection{Gravitational time delay}

An interesting relativistic effect in the propagation of light rays is the apparent delay in the time of propagation for a light signal passing near the Sun, which is a relevant correction for astronomic observations, and is called the Shapiro time delay. The time delay of radar echo corresponds to the determination of the time delay of radar signals which are transmitted from the Earth through a region near the Sun to another planet or spacecraft and then reflected back to the Earth. The time interval between emission and return of a pulse as measured by a clock on the Earth is

$t_{12}=2 t\left(r_{1}, \rho_{0}\right)+2 t\left(r_{2}, \rho_{0}\right)$, where $\rho_{0}$ is for the closest approach to the Sun. Now, in order to calculate the time delay we use (18), (21) and the coordinate time

$\dot{r}=\dot{t} \frac{\mathrm{d} r}{\mathrm{~d} t}=\frac{E}{F(r)} \frac{\mathrm{d} r}{\mathrm{~d} t}$

so (16) can be written as

$\frac{E}{F(r)} \frac{\mathrm{d} r}{\mathrm{~d} t}=\sqrt{E^{2}-\frac{L^{2}}{r^{2}} F(r)}$.

By considering $\rho_{0}$ as the closest approach to the Sun, $d r / d t$ vanishes, so that

$\frac{E^{2}}{L^{2}}=\frac{F\left(\rho_{0}\right)}{\rho_{0}^{2}}$.

Now, by inserting (55) in (54), the coordinate time which the light requires to go from $\rho_{0}$ to $r$ is

$t\left(r, \rho_{0}\right)=\int_{\rho_{0}}^{r} \frac{\mathrm{d} r}{F(r) \sqrt{1-\frac{\rho_{0}^{2}}{F\left(\rho_{0}\right)} \frac{F(r)}{r^{2}}}}$.

So, at first order correction we obtain

$$
\begin{aligned}
t\left(r, \rho_{0}\right)= & \sqrt{r^{2}-\rho_{0}^{2}}+\frac{M}{m_{\mathrm{p}}^{2}} \\
& \times\left[\sqrt{\frac{r-\rho_{0}}{r+\rho_{0}}}+2 \ln \left(\frac{r+\sqrt{r^{2}-\rho_{0}^{2}}}{\rho_{0}}\right)\right]+ \\
& +\frac{m_{\mathrm{p}}^{4}}{300 z^{2}} \sqrt{r^{2}-\rho_{0}^{2}}\left[\frac{9 \rho_{0}^{2}}{2}-\frac{3 \rho_{0}^{2} r^{2}}{2}-3 r^{4}\right] .
\end{aligned}
$$

Therefore, for the circuit from point 1 to point 2 and back the delay in the coordinate time is

$$
\begin{aligned}
\Delta t: & =2\left[t\left(r_{1}, \rho_{0}\right)+t\left(r_{2}, \rho_{0}\right)-\sqrt{r_{1}^{2}-\rho_{0}^{2}}-\sqrt{r_{2}^{2}-\rho_{0}^{2}}\right] \\
& =\Delta t_{M}+\Delta t_{z}
\end{aligned}
$$

where

$$
\begin{aligned}
\Delta t_{M}= & \frac{2 M}{m_{\mathrm{p}}^{2}}\left[2 \ln \left(\frac{\left(r_{1}+\sqrt{r_{1}^{2}-\rho_{0}^{2}}\right)\left(r_{2}+\sqrt{r_{2}^{2}-\rho_{0}^{2}}\right)}{\rho_{0}^{2}}\right)\right. \\
& \left.+\sqrt{\frac{r_{1}-\rho_{0}}{r_{1}+\rho_{0}}}+\sqrt{\frac{r_{2}-\rho_{0}}{r_{2}+\rho_{0}}}\right] \\
\Delta t_{z}= & \frac{m_{\mathrm{p}}^{4}}{300 z^{2}}\left[\sqrt{r_{1}^{2}-\rho_{0}^{2}}\left(\frac{9 \rho_{0}^{2}}{2}-\frac{3 \rho_{0}^{2} r_{1}^{2}}{2}-3 r_{1}^{4}\right)\right. \\
& \left.+\sqrt{r_{2}^{2}-\rho_{0}^{2}}\left(\frac{9 \rho_{0}^{2}}{2}-\frac{3 \rho_{0}^{2} r_{2}^{2}}{2}-3 r_{2}^{4}\right)\right]
\end{aligned}
$$


For a round trip in the solar system, we have $\left(\rho_{0}<<\right.$ $\left.r_{1}, r_{2}\right)$

$$
\begin{aligned}
\Delta t & \approx \frac{4 M}{m_{\mathrm{p}}^{2}}\left[1+\ln \left(\frac{4 r_{1} r_{2}}{\rho_{0}^{2}}\right)\right]-\frac{m_{\mathrm{p}}^{4}}{100 z^{2}}\left(r_{1}^{5}+r_{2}^{5}\right) \\
& =\Delta t_{\mathrm{GR}}+\Delta t_{\text {Horndeski }} .
\end{aligned}
$$

Note that, if we consider the limit $\frac{M}{m_{\mathrm{p}}^{2}} \rightarrow M_{\odot}$ and $z \rightarrow \infty$, we recover the classical result of GR; that is, $\Delta t_{\mathrm{GR}}=4 M_{\odot}\left[1+\ln \left(\frac{4 r_{1} r_{2}}{\rho_{0}^{2}}\right)\right]$. For a round trip from the Earth to Mars and back, we get (for $\rho_{0} \ll r_{1}, r_{2}$ ), where $r_{1} \approx r_{2}=2.25 \times 10^{11} \mathrm{~m}$ is the average Earth-Mars distance. Considering $\rho_{0}$ as the point of closest approach to the Sun, like the radius of the Sun $\left(R_{\odot} \approx 6.960 \times 10^{8} \mathrm{~m}\right)$ plus the solar corona $\left(\sim 10^{9} \mathrm{~m}\right), \rho_{0} \approx 1.696 \times 10^{9} \mathrm{~m}$, then the time delay is $\Delta t_{\mathrm{GR}} \approx 240 \mu \mathrm{s}$. To give an idea of the experimental possibilities, we mention that the error in the time measurement of a circuit during the Viking mission was only about $10 \mathrm{~ns}$ [68]. If the Horndeski term contributes, $\Delta t_{\text {Horndeski }}=-\frac{m_{\mathrm{p}}^{4}}{100 z^{2}}\left(r_{1}^{5}+r_{2}^{5}\right)$, it does so in such a way that $\Delta t_{\text {Horndeski }}<10^{-8} \mathrm{~s}$, or $\sqrt{z} / m_{\mathrm{p}}>7.9 \times 10^{12}(\mathrm{~m})$.

\section{Concluding comments}

We considered four-dimensional Horndeski black holes and we analyzed the motion of particles in these backgrounds with the objective to study the geodesics and to constrain the value of the derivative coupling parameter $z$ through solar system tests.

Concerning the radial and angular time-like geodesics, we found that the motion of particles is confined, while for the Schwarzschild spacetime the radial and angular timelike geodesics are confined but there are also unconfined geodesics. However, both spacetimes allow for the existence of stable and unstable circular orbits, as well as the existence of planetary orbits. For null geodesics, four-dimensional Horndeski and Schwarzschild spacetimes allow for the existence of a capture zone, an unstable circular orbit, and a deflection zone. However, for Schwarzschild spacetime the effective potential vanishes at infinity, while for Horndeski spacetime the effective potential goes to $\frac{L^{2} m p^{2}}{12 z}$ at infinity; thus, for $z \rightarrow \infty$ we recover GR. In this way, the behavior of the geodesics is qualitatively similar to the behavior of the geodesics in a Schwarzschild AdS spacetime [36].

So as to constrain the value of the $z$ parameter through solar system tests, we considered the perihelion precession, the gravitational redshift, the deflection of light and the gravitational time delay. Our results show that it is possible to constrain the value of the derivative coupling parameter $z$ $\left(\sqrt{z} / m_{\mathrm{p}}>2.6 \times 10^{12} \mathrm{~m}\right)$ in agreement with all the observational tests that have been considered.

We found that the perihelion advance has the standard value of GR plus the correction term coming from Horndeski's theory, given by $\Delta \tilde{\phi} \approx 2 \pi+\frac{6 \pi M}{m_{\mathrm{p}}^{2} l}-\frac{\pi m_{\mathrm{p}}^{4} l^{4}}{15 z^{2}}$; thus, we constrained the $z$ parameter to $\sqrt{z} / m_{\mathrm{p}} \geq 2.6 \times 10^{12} \mathrm{~m}$, in order to obtain a better accuracy between the observational value and the theoretical value of the precession of perihelion for Mercury.

Also, we obtained the result that the gravitational redshift is $\frac{v}{v_{0}} \approx 1+\frac{M}{m_{\mathrm{p}}^{2} r_{0}}-\frac{M}{m_{\mathrm{p}}^{2} r}+\frac{r^{4} m_{\mathrm{p}}^{4}}{40 z^{2}}-\frac{r_{0}^{4} m_{\mathrm{p}}^{4}}{40 z^{2}}$, and in the limit $\frac{M}{m_{\mathrm{p}}^{2}} \rightarrow M_{\oplus}$ and $z \rightarrow \infty$, the classical result for the Schwarzschild spacetime is recovered; thus, by considering that this observation is well described within Einstein's theory, the extra Horndeski terms must be $<10^{-14}$, which implies the constraint $\frac{\sqrt{z}}{m_{\mathrm{p}}}>2.7 \times 10^{10} \mathrm{~m}$.

The deflection of light is given by $\hat{\alpha}=\frac{4 M}{m_{\mathrm{p}}^{2} b}+\frac{m_{\mathrm{p}}^{4} b^{4} \sqrt{2}}{10 z^{2}}$, and in the limit $\frac{M}{m_{\mathrm{p}}^{2}} \rightarrow M_{\odot}$ and $z \rightarrow \infty$, we recovered the classical result of GR; that is, $\hat{\alpha}_{\mathrm{GR}}=4 M_{\odot} / b$. In this case, we have shown that there is a zone where the deflection is attractive and another zone where the deflection is repulsive. Also, the observational values compared to the classic result are smaller, and the contribution of the Horndeski term to the deflection angle is positive; therefore, there is no observable effect. Thus, if the Horndeski term contributes it does so in such a way that $\hat{\alpha}_{\text {Horndeski }}<0.00001^{\prime \prime}$, or $\sqrt{z} / m_{\mathrm{p}}>$ $1.6 \times 10^{11} \mathrm{~m}$.

Finally, as regards the gravitational time delay for a round trip in the solar system, we found that $\Delta t=\Delta t_{\mathrm{GR}}+$ $\Delta t_{\mathrm{Horndeski}}$, where $\Delta t_{\mathrm{GR}}=4 M_{\odot}\left[1+\ln \left(\frac{4 r_{1} r_{2}}{\rho_{0}^{2}}\right)\right]$, and $\Delta t_{\text {Horndeski }}=-\frac{m_{\mathrm{p}}^{4}}{100 z^{2}}\left(r_{1}^{5}+r_{2}^{5}\right)$; thus, in the limit $\frac{M}{m_{\mathrm{p}}^{2}} \rightarrow M_{\odot}$ and $z \rightarrow \infty$, we recover the classical result of GR; for a round trip from the Earth to Mars and back, the time delay is $\Delta t_{\mathrm{GR}} \approx 240 \mu \mathrm{s}$. However, the error in the time measurement of a circuit during the Viking mission was only about $10 \mathrm{~ns}$ [68]. Therefore, if the Horndeski term contributes, it does so for $\Delta t_{\text {Horndeski }}<10^{-8} \mathrm{~s}$, or $\sqrt{z} / m_{\mathrm{p}}>7.9 \times 10^{12} \mathrm{~m}$.

Acknowledgements We thank the referee for his/her careful review of the manuscript and his/her valuable comments and suggestions which helped us to improve the manuscript. We thank Eugeny Babichev and Shinji Tsujikawa for their valuable comments and remarks. Y.V. acknowledges support by the Dirección de Investigación y Desarrollo de la Universidad de La Serena, Grant No. PR18142.

Data Availability Statement This manuscript has no associated data or the data will not be deposited. [Authors' comment: This is a theoretical paper without associated data.] 
Open Access This article is licensed under a Creative Commons Attribution 4.0 International License, which permits use, sharing, adaptation, distribution and reproduction in any medium or format, as long as you give appropriate credit to the original author(s) and the source, provide a link to the Creative Commons licence, and indicate if changes were made. The images or other third party material in this article are included in the article's Creative Commons licence, unless indicated otherwise in a credit line to the material. If material is not included in the article's Creative Commons licence and your intended use is not permitted by statutory regulation or exceeds the permitted use, you will need to obtain permission directly from the copyright holder. To view a copy of this licence, visit http://creativecomm ons.org/licenses/by/4.0/.

Funded by SCOAP ${ }^{3}$.

\section{References}

1. LIGO Scientific and Virgo Collaborations Collaboration, B.P. Abbott et al., Observation of gravitational waves from a binary black hole merger. Phys. Rev. Lett. 116, 061102 (2016)

2. VGW151226: observation of gravitational waves from a 22-solarmass binary black hole coalescence. Phys. Rev. Lett. 116, 241103 (2016)

3. VIRGO, LIGO Scientific Collaboration, B.P. Abbott et al., GW170104: observation of a 50-solar-mass binary black hole coalescence at redshift 0.2. Phys. Rev. Lett. 118, 221101 (2017)

4. Virgo, LIGO Scientific Collaboration, B.P. Abbott et al., GW170814: a three-detector observation of gravitational waves from a binary black hole coalescence. Phys. Rev. Lett. 119, 141101 (2017)

5. Virgo, LIGO Scientific Collaboration, B.P. Abbott et al., GW170817: observation of gravitational waves from a binary neutron star inspiral. Phys. Rev. Lett. 119, 161101 (2017)

6. Y.Fujii, K. Maeda, The Scalar-Tensor Theory of Gravitation (Cambridge University Press, Cambridge, 2007)

7. G.W. Horndeski, Second-order scalar-tensor field equations in a four-dimensional space. Int. J. Theor. Phys. 10, 363 (1974)

8. M. Ostrogradsky, Mémoires sur les équations différentielles, relatives au problème des isopérimètres. Mem. Acad. St. Petersbourg 6(4), 385 (1850)

9. A. Nicolis, R. Rattazzi, E. Trincherini, The Galileon as a local modification of gravity. Phys. Rev. D 79, 064036 (2009)

10. C. Deffayet, G. Esposito-Farese, A. Vikman, Covariant Galileon. Phys. Rev. D 79, 084003 (2009)

11. T. Kolyvaris, G. Koutsoumbas, E. Papantonopoulos, G. Siopsis, Scalar hair from a derivative coupling of a scalar field to the Einstein tensor. Class. Quantum Gravity 29, 205011 (2012)

12. M. Rinaldi, Black holes with non-minimal derivative coupling. Phys. Rev. D 86, 084048 (2012). arXiv:1208.0103 [gr-qc]

13. T. Kolyvaris, G. Koutsoumbas, E. Papantonopoulos, G. Siopsis, Phase transition to a hairy black hole in asymptotically flat spacetime. JHEP 11, 133 (2013)

14. E. Babichev, C. Charmousis, Dressing a black hole with a timedependent Galileon. JHEP 08, 106 (2014)

15. C. Charmousis, T. Kolyvaris, E. Papantonopoulos, M. Tsoukalas, Black holes in bi-scalar extensions of Horndeski theories. JHEP 07, 085 (2014)

16. L. Amendola, Cosmology with nonminimal derivative couplings. Phys. Lett. B 301, 175 (1993). arXiv:gr-qc/9302010

17. S.V. Sushkov, Exact cosmological solutions with nonminimal derivative coupling. Phys. Rev. D 80, 103505 (2009). arXiv:0910.0980 [gr-qc]

18. C. Germani, A. Kehagias, UV-protected inflation. Phys. Rev. Lett. 106, 161302 (2011)
19. E.N. Saridakis, S.V. Sushkov, Quintessence and phantom cosmology with non-minimal derivative coupling. Phys. Rev. D 81, $083510(2010)$

20. Y. Huang, Q. Gao, Y. Gong, The phase-space analysis of scalar fields with non-minimally derivative coupling. Eur. J. Phys. C 75, 143 (2015)

21. N. Yang, Q. Fei, Q. Gao, Y. Gong, Inflationary models with nonminimally derivative coupling. Class. Quantum Gravity 33(20), 205001 (2016)

22. G. Koutsoumbas, K. Ntrekis, E. Papantonopoulos, Gravitational particle production in gravity theories with non-minimal derivative couplings. JCAP 08, 027 (2013)

23. C. Germani, A. Kehagias, New model of inflation with nonminimal derivative coupling of standard model Higgs boson to gravity. Phys. Rev. Lett. 105, 011302 (2010)

24. C. Germani, Y. Watanabe, UV-protected (natural) inflation: primordial fluctuations and non-Gaussian features. JCAP 1107, 031 (2011) [Addendum: JCAP 1107, A01 (2011)]

25. L. Lombriser, A. Taylor, Breaking a dark degeneracy with gravitational waves. JCAP 1603(03), 031 (2016)

26. L. Lombriser, N.A. Lima, Challenges to self-acceleration in modified gravity from gravitational waves and large-scale structure. Phys. Lett. B 765, 382 (2017)

27. D. Bettoni, J.M. Ezquiaga, K. Hinterbichler, M. Zumalacárregui, Speed of gravitational waves and the fate of scalar-tensor gravity. Phys. Rev. D 95(8), 084029 (2017)

28. T. Baker, E. Bellini, P.G. Ferreira, M. Lagos, J. Noller, I. Sawicki, Strong constraints on cosmological gravity from GW170817 and GRB 170817A. Phys. Rev. Lett. 119(25), 251301 (2017). arXiv: 1710.06394 [astro-ph.CO]

29. P. Creminelli, F. Vernizzi, Dark energy after GW170817 and GRB170817A. Phys. Rev. Lett. 119(25), 251302 (2017). arXiv:1710.05877 [astro-ph.CO]

30. J. Sakstein, B. Jain, Implications of the neutron star merger GW170817 for cosmological scalar-tensor theories. Phys. Rev. Lett. 119(25), 251303 (2017). arXiv:1710.05893 [astro-ph.CO]

31. J.M. Ezquiaga, M. Zumalacárregui, Phys. Rev. Lett. 119(25), 251304 (2017). arXiv:1710.05901 [astro-ph.CO]

32. B.P. Abbott et al. (LIGO Scientific, Virgo, Fermi-GBM and INTEGRAL), Gravitational waves and gamma-rays from a binary neutron star merger: GW170817 and GRB 170817A. Astrophys. J. Lett. 848(2), L13 (2017). arXiv:1710.05834 [astro-ph.HE]

33. C. Deffayet, S. Deser, G. Esposito-Farese, Generalized Galileons: all scalar models whose curved background extensions maintain second-order field equations and stress-tensors. Phys. Rev. D 80, 064015 (2009). arXiv:0906.1967 [gr-qc]

34. Y. Gong, E. Papantonopoulos, Z. Yi, Constraints on scalar-tensor theory of gravity by the recent observational results on gravitational waves. Eur. Phys. J. C 78(9), 738 (2018). arXiv:1711.04102 [gr-qc]

35. S. Chakraborty, S. SenGupta, Solar system constraints on alternative gravity theories. Phys. Rev. D 89(2), 026003 (2014)

36. N. Cruz, M. Olivares, J.R. Villanueva, The Geodesic structure of the Schwarzschild anti-de Sitter black hole. Class. Quantum Gravity 22, 1167 (2005). arXiv:gr-qc/0408016

37. M. Vasudevan, K.A. Stevens, Integrability of particle motion and scalar field propagation in Kerr-(anti) de Sitter black hole spacetimes in all dimensions. Phys. Rev. D 72, 124008 (2005). arXiv:gr-qc/0507096

38. E. Hackmann, C. Lammerzahl, Geodesic equation in Schwarzschild- (anti-) de Sitter space-times: analytical solutions and applications. Phys. Rev. D 78, 024035 (2008). arXiv: 1505.07973 [gr-qc]

39. E. Hackmann, C. Lammerzahl, Complete analytic solution of the geodesic equation in Schwarzschild- (anti-) de Sitter spacetimes. Phys. Rev. Lett. 100, 171101 (2008). arXiv:1505.07955 [gr-qc] 
40. M. Olivares, J. Saavedra, J.R. Villanueva, C. Leiva, Motion of charged particles on the Reissner-Nordstróm (anti)-de Sitter black holes. Mod. Phys. Lett. A 26, 2923 (2011). arXiv:1101.0748 [grqc]

41. N. Cruz, M. Olivares, J. Saavedra, J.R. Villanueva, Null geodesics in the Reissner-Nordstrom anti-de Sitter black holes. arXiv:1111.0924 [gr-qc]

42. A. Larranaga, Geodesic structure of the noncommutative Schwarzschild anti-de Sitter black hole I: timelike geodesics. Rom. J. Phys. 58, 50 (2013). arXiv:1110.0778 [gr-qc]

43. J.R. Villanueva, J. Saavedra, M. Olivares, N. Cruz, Photons motion in charged anti-de Sitter black holes. Astrophys. Space Sci. 344, 437 (2013)

44. P.A. Gonzalez, E. Papantonopoulos, J. Saavedra, Y. Vasquez, Fourdimensional asymptotically AdS black holes with scalar hair. JHEP 1312, 021 (2013). arXiv:1309.2161 [gr-qc]

45. P.A. Gonzalez, M. Olivares, Y. Vasquez, Motion of particles on a four-dimensional asymptotically AdS black hole with scalar hair. Eur. Phys. J. C 75(10), 464 (2015). arXiv:1507.03610 [gr-qc]

46. S. Bhattacharya, S. Chakraborty, Constraining some Horndeski gravity theories. Phys. Rev. D 95(4), 044037 (2017)

47. A.I. Vainshtein, To the problem of nonvanishing gravitation mass. Phys. Lett. B 39, 393-394 (1972)

48. E. Babichev, C. Deffayet, An introduction to the Vainshtein mechanism. Class. Quant. Grav. 30, 184001 (2013). https://doi.org/10. 1088/0264-9381/30/18/184001. arXiv:1304.7240 [gr-qc]

49. K. Koyama, G. Niz, G. Tasinato, Analytic solutions in nonlinear massive gravity. Phys. Rev. Lett. 107, 131101 (2011). arXiv:1103.4708 [hep-th]

50. K. Koyama, G. Niz, G. Tasinato, Strong interactions and exact solutions in non-linear massive gravity. Phys. Rev. D 84, 064033 (2011). arXiv:1104.2143 [hep-th]

51. K. Koyama, G. Niz, G. Tasinato, Effective theory for the Vainshtein mechanism from the Horndeski action. Phys. Rev. D 88, 021502 (2013). arXiv:1305.0279 [hep-th]

52. R. Kimura, T. Kobayashi, K. Yamamoto, Vainshtein screening in a cosmological background in the most general second-order scalartensor theory. Phys. Rev. D 85, 024023 (2012). arXiv:1111.6749 [astro-ph.CO]

53. A. De Felice, R. Kase, S. Tsujikawa, Vainshtein mechanism in second-order scalar-tensor theories. Phys. Rev. D 85, 044059 (2012)
54. R. Kase, S. Tsujikawa, Screening the fifth force in the Horndeski's most general scalar-tensor theories. JCAP 1308, 054 (2013). arXiv:1306.6401 [gr-qc]

55. P.A. González, M. Olivares, Y. Vásquez, J. Saavedra, A. Övgün, Motion and collision of particles near DST Black holes. Eur. Phys. J. C 79(6), 528 (2019). arXiv:1811.08551 [gr-qc]

56. M. Olivares, J.R. Villanueva, Massive neutral particles on heterotic string theory. Eur. Phys. J. C 73, 2659 (2013). arXiv:1311.4236 [grqc]

57. S. Chandrasekhar, The Mathematical Theory of Black Holes (Oxford University Press, New York, 1983)

58. S. Cornbleet, Am. J. Phys. 61, 650 (1993)

59. G.M. Clemence, The relativity effect in planetary motions. Rev. Mod. Phys. 19(4), 361-364 (1947)

60. NASA Jet Propulsion Laboratory. http://ssd.jpl.nasa.gov/? constants

61. R.A. Matzner, Dictionary of Geophysics, Astrophysics, and Astronomy (CRC Press, Boca Raton, 2001), p. 356. ISBN:0849328918

62. L. Iorio, On the possibility of measuring the solar oblateness and some relativistic effects from planetary ranging. Astron. Astrophys. 433, 385 (2005). arXiv:gr-qc/0406041

63. G.V. Kraniotis, S.B. Whitehouse, Compact calculation of the perihelion precession of Mercury in general relativity, the cosmological constant and Jacobi's inversion problem. Class. Quantum Gravity 20, 4817-4835 (2003). arXiv:astro-ph/0305181

64. C.M. Will, Theory and Experiment in Gravitational Physics, revised edn. (Cambridge University Press, Cambridge, 1993)

65. R.M. Wald, General Relativity (The University Chicago Press, Chicago, 1984)

66. R.F.C. Vessot et al., Test of relativistic gravitation with a spaceborne hydrogen maser. Phys. Rev. Lett. 45, 2081 (1980)

67. V. Kagramanova, J. Kunz, C. Lammerzahl, Solar system effects in Schwarzschild-de Sitter spacetime. Phys. Lett. B 634, 465 (2006). arXiv:gr-qc/0602002

68. N. Straumann, General Relativity and Relativistic Astrophysics (Springer, Berlin, 1984)

69. S.S. Shapiro, J.L. Davis, D.E. Lebach, J.S. Gregory, Measurement of the solar gravitational deflection of radio waves using geodetic very-long-baseline interferometry data, 1979-1999. Phys. Rev. Lett. 92, 121101 (2004) 